\title{
ASYMPTOTIC RESULTS OF A RECURSIVE DOUBLE KERNEL ESTIMATOR OF THE CONDITIONAL QUANTILE FOR FUNCTIONAL ERGODIC DATA
}

\author{
IMANE BOUAZZA $^{a}$, FATIMA BENZIADI $^{b}, \mathrm{FETHI} \mathrm{MADANI}^{c}$ \\ AND TOUFIK GUENDOUZI ${ }^{d}$
}

\author{
Laboratory of Stochastic Models, Statistics and Applications, University of Saida-Dr. Moulay Tahar, \\ P.O. Box 138, EN-NASR, 20000, Algeria. \\ ${ }^{a}$ E-mail: imen.bouazza@univ-saida.dz \\ ${ }^{b}$ E-mail: benziadi.fatima@univ-saida.dz \\ ${ }^{c}$ E-mail: fethi.madani@univ-saida.dz \\ ${ }^{d}$ E-mail: toufik.guendouzi@univ-saida.dz \\ ||\|
}

\begin{abstract}
The aim of our paper is to investigate the estimation of conditional quantile of a scalar response variable $Y$ given a random variable (rv) $X=x$ taking values in a semimetric space. Hence, the asymptotic normality of the proposed estimator is obtained when the observations are sampled from a functional ergodic process. The result confirms the prospect proposed in Benziadi et al. 3] and as applications, a comparison study based on a finite-sample behavior of the estimator is investigated by simulations as well.
\end{abstract}

\section{Introduction}

In nonparametric statistics, the estimation of conditional quantiles is becoming increasingly an important problem which has been widely studied because of their importance in several applications such as agronomy, medicine, economic ... and so on. Historically, countless works have been documented on this problem: By a direct method, the estimator of conditional quantile $t_{\alpha}(x)$ was proposed and then studied firstly by Koenker and

Received July 24, 2021.

AMS Subject Classification: Primary, 62G20; Secondary 62G08, 62G35, 62E20.

Key words and phrases: Asymptotic normality, conditional quantile, recursive estimate, ergodic data, functional data, small ball probability. 
Bassett [15] and defined as follow

$$
\widehat{t}_{\alpha}(x)=\arg \min _{t_{\alpha}} \sum_{i=1}^{n} \rho_{\alpha}\left(Y_{i}-t_{\alpha}\right), \quad \text { for }(x, y) \in \mathbb{R}^{2}
$$

Which made many scholars invest in this topic, among whom Cardot et al. [5], Koenker [14]. Subsequently, in the event that the observations are functional, Gannoun et al. [13] proposed a nonparametric conditional median predictors based on the double kernel method. The asymptotic properties of no-parametric conditional quantile estimator was established by Ezzahrioui and Ould-Saïd [10], Laksaci et al. [17] and studied the almost complete consistency and the asymptotic normality of a generalized $L^{1}$-approach for a kernel estimator of conditional quantile with functional regressor.

However, studies of conditional quantile estimation are a significant subject that has given rise to a large number of contributions and their application is very wide and covers various fields, often involve both prediction setting and in estimation of regression function. Several works on the regression quantile exist in the literature, the first idea for this subject, was proposed by Stone [20]. Cardot et al. [5] have proved the $L^{2}$-convergence rate of the conditional quantile as a linear regression model for functional data. Their results have been extended to the kernel case by Ferraty et al. 11] who have proposed a nonparametric estimator of this model and they have established the almost complete convergence for the i.i.d case. On the other hand, the convergence in $L^{p}$-norm stated by Dabo-Niang and Laksaci [8]. The interested reader can also refer to some of the following additional references [18], [22] and [7] to expand further on this topic and take an overview.

Otherwise, considering the recursive conditional models estimate, literary, the first result in this topic was developed by Wolverton and Wagner [21], they had established the asymptotically optimal discriminant functions for pattern classification. Masry and Györfi [19] was also treated for weakly dependence stationary process the recursive estimator of probability density. Recently, Amiri [1] has investigated the asymptotic properties of the recursive regression estimator with application in the non-parametric prediction. In the same aim, the almost sure convergence rates of the conditional geometric median estimator proved by Cardot et al. [6] in Hilbert space. Amiri 
et al. [2] obtained the asymptotic properties of the recursive estimator of the regression function with functional covariate.

In this present work, in regard to the dependence setting, our focus is to use ergodic variables to allow the maximum possible generality and to estimate the conditional quantile function in this case. Note here that, the nonparametric kernel regression estimation for functional stationary ergodic data was considered by Laib and Louani [16], they have studied the consistency in probability, with a rate, as well as the asymptotic normality of this estimator. Also, for i.i.d functional data, Bouadjemi [4] have established the asymptotic normality for the conditional cumulative distribution function. More recently, Benziadi et al. [3] have studied the almost complete (a.c) convergence with rates of the functional recursive kernel estimate of the conditional quantile.

The outline of this paper is described as follows: first, we define the double-kernel recursive estimator when the covariate $X$ is functional in Section 2. We establish then the asymptotic normality of this model as well as the confidence interval in Section 4 and 5 under the assumptions given in Section 3. A computational study is carried out to evaluate and understand how effective this resulting model is, in Section 6. Finally, in the Section 7 devoted to appendix, we present the detailed proofs of the auxiliary results. Indeed, in the setting of ergodic processes, to prove our results, our methodology is based mainly on the martingale approximation which allows to launch a systematic study for dependent data.

\section{Definition of the Model}

To fix notation, let $\left(X_{i}, Y_{i}\right)_{i=1, \ldots, n}$ be a sequence of strictly stationary dependent random variables valued in $\mathcal{F} \times \mathbb{R}$ and observable from the same subject, where $\mathcal{F}$ is a semi-metric space. For $\alpha \in[0,1]$, the conditional quantile of order $\alpha$ defined by the inverse of the conditional distribution function $F^{x}(y)$ of $Y$ given $X=x$ is denoted $t_{\alpha}(x)$. To estimate it, we use the estimator of the nonparametric function given usually by:

$$
F^{x}(y)=\mathbb{P}[Y \leq y / X=x]
$$


Thus, based on the finding proof of Laksaci et al. [17], we note that this estimator can be given by:

$$
\widehat{t}_{\alpha}(x)=\inf \left\{y \in \mathbb{R} / \widehat{F}^{x}(y)=\alpha\right\}
$$

In the sequel, let $\widehat{F}^{x}(y)$ be the recursive estimate of $F^{x}(y)$ defined as:

$$
\widehat{F}^{x}(y)=\frac{\sum_{i=1}^{n} K\left(a_{i}^{-1} d\left(x, X_{i}\right)\right) H\left(b_{i}^{-1}\left(y-Y_{i}\right)\right)}{\sum_{i=1}^{n} K\left(a_{i}^{-1} d\left(x, X_{i}\right)\right)}, \quad \forall y \in \mathbb{R}
$$

where $K$ is a kernel strictly increasing distribution defined for $x \in \mathcal{F}$ and $\left(a_{i}\right)$ $\left(\right.$ resp $\left.\left(b_{i}\right)\right)$ is a sequence of positive real numbers such that $\lim _{n \rightarrow \infty} a_{n}=0$ resp $\left(\lim _{n \longrightarrow \infty} b_{n}=0\right)$. The main advantage of this estimation method is to update the estimate for each additional observation without resorting to past data.

\section{General Framework and Assumptions}

The general framework of our contribution is the nonparametric modeling in functional ergodic data. To this aim, in such study, we formulate these data by the following notations, for all $k=1, \cdots, n$, we introduce $\mathfrak{F}_{k}$ the $\sigma$-field generated by $\left(\left(X_{1}, Y_{1}\right), \cdots,\left(X_{k}, Y_{k}\right)\right)$ and $\mathfrak{B}_{k}$ the $\sigma$-field generated by $\left(\left(X_{1}, Y_{1}\right), \cdots,\left(X_{k}, Y_{k}\right), X_{k+1}\right)$. In addition, $x$ will stand from now on for a fixed point in $\mathcal{F}$ and $C$ or $C^{\prime}$ denote some generic constant in $\mathbb{R}^{*+}$, and $N_{x}$ denote the fixed neighborhood of $x$.

Therefore, our asymptotic results are stated under the following assumptions of our model that we gathered hereafter for easy reference. 
(H1) The strictly stationary ergodic process $\left(X_{i}, Y_{i}\right)_{i \in \mathbb{N}^{*}}$ satisfies:

(i) The function $\phi(x, r):=\mathbb{P}(X \in B(x, r))$ is such that $\phi(x, r)>0$, $\forall r>0$ where $B(x, h)=\left\{x^{\prime} \in \mathcal{F} / d\left(x^{\prime}, x\right)<h\right\}$.

(ii) For all $i=1, \ldots, n$ there exists a deterministic function, $\phi_{i}(x, \cdot)$ such that almost surely $0<\mathbb{P}\left(X_{i} \in B(x, r) \mid \mathfrak{F}_{i-1}\right) \leq \phi_{i}(x, r)$, $\forall r>0$, and $\phi_{i}(x, r) \rightarrow 0$ as $r \rightarrow 0$.

(iii) For all sequence $\left(r_{i}\right)_{i=1, \ldots n}>0$,

$$
\frac{\sum_{i=1}^{n} \mathbb{P}\left(X_{i} \in B\left(x, r_{i}\right) \mid \mathfrak{F}_{i-1}\right)}{\sum_{i=1}^{n} \phi\left(x, r_{i}\right)} \rightarrow 1 \quad \text { a.co. }
$$

For a fixed neighborhood $N_{x}$ of $x$, we assume that the regular version $F^{x^{\prime}}$ of the conditional distribution function of $Y$ given $X=x^{\prime}$ exists for all $x^{\prime} \in N_{x}$ and we suppose that $F^{x}$ has a continuous density $f^{x}$ with respect to (w.r.t.) Lebesgue measure over $\mathbb{R}$ and is such that

(H2) There exists $\delta>0$, such that $\forall\left(t_{1}, t_{2}\right) \in\left[t_{\alpha}(x)-\delta, t_{\alpha}(x)+\delta\right]^{2}$, $\forall\left(x_{1}, x_{2}\right) \in N_{x}^{2}, \forall y \in \mathbb{R}$

$$
\left|F^{x_{1}}\left(t_{1}\right)-F^{x_{2}}\left(t_{2}\right)\right| \leq C^{\prime}\left(d\left(x_{1}, x_{2}\right)^{\beta_{1}}+\left|t_{1}-t_{2}\right|^{\beta_{2}}\right)
$$

and for $C, C^{\prime}, \beta_{1}$ and $\beta_{2} \in \mathbb{R}^{*+}$

$$
\inf _{y \in\left[t_{\alpha}(x)-\delta, t_{\alpha}(x)+\delta\right]} f^{x}(y)>C
$$

(H3) The positive bandwidths $\left(a_{i}, b_{i}\right)$ satisfying: $\forall t \in[0,1]$

$$
\begin{aligned}
& \lim _{n \rightarrow \infty} \frac{\phi\left(x, t a_{n}\right)}{\phi\left(x, a_{n}\right)}=\beta_{x}(t) \\
& \frac{1}{n \varphi_{n}(x)} \sum_{i=1}^{n}\left(\left(c a_{i}^{\beta_{1}}+c^{\prime} b_{i}^{\beta_{2}}\right) \phi\left(x, a_{i}\right)\right)^{2} \longrightarrow 0 \text { as } n \longrightarrow \infty
\end{aligned}
$$

and

$$
n \varphi_{n}(x) \longrightarrow \infty \text { with } \varphi_{n}(x)=n^{-1} \sum_{i=1}^{n} \phi\left(x, a_{i}\right)
$$


(H4) $K$ is a function and having a compact support on $(0,1)$, such that:

$$
C \mathbb{I}_{(0,1)}<K(t)<C^{\prime} \mathbb{I}_{(0,1)}
$$

(H5) The function $H$ is of class $\mathcal{C}^{1}$ such that

$$
\left\{\begin{array}{l}
\forall\left(y_{1}, y_{2}\right) \in \mathbb{R}^{2},\left|H\left(y_{1}\right)-H\left(y_{2}\right)\right| \leq C\left|y_{1}-y_{2}\right| \\
\int|t|^{\beta_{2}} H^{(1)}(t) d t<\infty, \text { where } \beta_{2} \text { is given in (H2). }
\end{array}\right.
$$

\subsection{Comments on the assumptions}

For our model, these assumptions are very usual for estimating the conditional quantile; condition (H1)(i) characterizes the property of concentration on small balls of the probability measure of the underlying explanatory variable. The ergodicity of functional data in assumption (H1)(ii) is the same as that classically imposed by Laib and Louani [16] for infinite-dimensional settings. The assumption (H2) ensure the regularity version $F^{x^{\prime}}$ of the conditional distribution function of $Y$ given $X=x^{\prime}$ for a fixed neighborhood $N_{x}$ of $x$, although there are several ways to define this nonparametric concept. For the semi-metric structure, this latter condition is more suitable. Condition (H3) plays a crucial role in the asymptotic normality result: In other words, the function $\beta_{x}$ is needed in the study of the variance term and (H4) is checked. In order to explicit asymptotically the bias term the condition (H5) is fulfilled.

\section{Main Results}

In the following, we announce the asymptotic results of the doublerecursive kernel estimator $\widehat{t}_{\alpha}(x)$ of $t_{\alpha}(x)$, where $\stackrel{D}{\longrightarrow}$ denotes the convergence in distribution and $\mathcal{N}(\cdot, \cdot)$ denotes the Gaussian distribution.

Proposition 1. Suppose that the conditions $(\mathrm{H} 1)-(\mathrm{H} 5)$ hold true. In addition, if the following condition is verified

$$
\lim _{n \rightarrow \infty} n \varphi_{n}(x)=\infty \quad \text { and } \quad \lim _{n \rightarrow \infty} \frac{\log n}{n \varphi_{n}(x)}=0,
$$


then,

$$
\left(\frac{n \varphi_{n}(x)}{\sigma^{2}(x)}\right)^{1 / 2}\left(\widehat{F}^{x}(y)-F^{x}(y)\right) \stackrel{D}{\longrightarrow} \mathcal{N}(0,1) \text { as } n \longrightarrow \infty .
$$

Theorem 1. Under the hypotheses of Proposition 1, we have for any $x \in \mathcal{F}$

$$
\left(\frac{n \varphi_{n}(x)}{\sigma^{2}(x)}\right)^{1 / 2}\left(\widehat{t}_{\alpha}(x)-t_{\alpha}(x)\right) \stackrel{D}{\longrightarrow} \mathcal{N}(0,1) \text { as } n \longrightarrow \infty
$$

where

$$
\sigma^{2}(x)=\left(\frac{\alpha(1-\alpha) \gamma_{1}}{\left(f^{x}\left(t_{\alpha}\right)\right)^{2} \gamma_{2}^{2}}\right)
$$

with

$\gamma_{1}=K^{2}(1)-\int_{0}^{1}\left(K^{2}(s)\right)^{\prime} \beta_{x}(s) d s>0, \quad \gamma_{2}=K(1)-\int_{0}^{1} K^{\prime}(s) \beta_{x}(s) d s \neq 0$ and $\mathcal{A}=\left\{x / \sigma^{2}(x) \neq 0\right\}$.

Proof of Proposition 1, We will rely on the following notation for the remainder of this paper: for any $(x, y) \in \mathcal{F} \times \mathbb{R}$ and $i=1, \cdots, n$

$$
K_{i}=K\left(a_{i}^{-1} d\left(x, X_{i}\right)\right) \text { and } H_{i}=H\left(b_{i}^{-1}\left(y-Y_{i}\right)\right)
$$

and then address the general decomposition used usually in this nonparametric case

$$
\widehat{F}^{x}(y)=\widehat{B}_{n}(x, y)+\frac{\widehat{R}_{n}(x, y)}{\widehat{F}_{D}(x)}+\frac{\widehat{Q}_{n}(x, y)}{\widehat{F}_{D}(x)}
$$

where

$$
\begin{aligned}
& \widehat{Q}_{n}(x, y):=\left(\widehat{F}_{N}^{x}(y)-\bar{F}_{N}^{x}(y)\right)-F^{x}(y)\left(\widehat{F}_{D}(x)-\bar{F}_{D}(x)\right) \\
& \widehat{B}_{n}(x, y):=\frac{\bar{F}_{N}^{x}(y)}{\bar{F}_{D}(x)} \text { and } \widehat{R}_{n}(x, y):=-\widehat{B}_{n}(x, y)\left(\widehat{F}_{D}(x)-\bar{F}_{D}(x)\right)
\end{aligned}
$$

with

$$
\widehat{F}_{N}^{x}(y):=\frac{1}{n \varphi_{n}} \sum_{i=1}^{n} K\left(a_{i}^{-1} d\left(x, X_{i}\right)\right) H\left(b_{i}^{-1}\left(y-Y_{i}\right)\right),
$$




$$
\begin{aligned}
& \bar{F}_{N}^{x}(y):=\frac{1}{n \varphi_{n}} \sum_{i=1}^{n} \mathbb{E}\left[K\left(a_{i}^{-1} d\left(x, X_{i}\right)\right) H\left(b_{i}^{-1}\left(y-Y_{i}\right)\right) \mid \mathfrak{F}_{i-1}\right] \\
& \widehat{F}_{D}(x):=\frac{1}{n \varphi_{n}} \sum_{i=1}^{n} K\left(a_{i}^{-1} d\left(x, X_{i}\right)\right) \\
& \bar{F}_{D}(x):=\frac{1}{n \varphi_{n}} \sum_{i=1}^{n} \mathbb{E}\left[K\left(a_{i}^{-1} d\left(x, X_{i}\right)\right) \mid \mathfrak{F}_{i-1}\right] .
\end{aligned}
$$

Thus, Proposition 1 is a consequence of the following intermediate results which proofs are given in the Appendix.

Lemma 1. Under hypotheses of Proposition 1, we have for any $x \in \mathcal{A}$

$$
\left(\frac{n \varphi_{n}(x) \gamma_{2}^{2}}{\gamma_{1} \alpha(1-\alpha)}\right)^{1 / 2} \widehat{Q}_{n}\left(x, \tau_{\alpha}(u, x)\right) \stackrel{D}{\longrightarrow} \mathcal{N}(0,1) \text { as } n \longrightarrow \infty .
$$

Lemma 2. Under hypotheses (H1) and (H4), we have:

$$
\widehat{F}_{D}(x)-1=\circ_{p}(1)
$$

Lemma 3. Under hypotheses (H1), (H2) and (H3), we have:

$$
\left(\frac{n \varphi_{n}(x) \gamma_{2}^{2}}{\gamma_{1} \alpha(1-\alpha)}\right)^{1 / 2} \widehat{B}_{n}\left(x, \tau_{\alpha}(u, x)\right)=u+\circ_{p}(1) \text { as } n \longrightarrow \infty \text {. }
$$

Lemma 4. Under hypotheses (H1), (H2) and (H4), we have

$$
\left(\frac{n \varphi_{n}(x) \gamma_{2}^{2}}{\gamma_{1} \alpha(1-\alpha)}\right)^{1 / 2} \widehat{R}_{n}\left(x, \tau_{\alpha}(u, x)\right)=\circ(1) \text { a.co as } n \longrightarrow \infty \text {. }
$$

Proof of Theorem 1. We define for all $u \in \mathbb{R}, \tau_{\alpha}(u, x)=t_{\alpha}(x)+$ $u\left[n \varphi_{n}(x)\right]^{-1 / 2} \sigma(x)$

$$
\begin{aligned}
\mathbb{P}\left\{\sqrt{n \varphi_{n}(x)} \sigma^{-1}(x)\left(\widehat{t}_{\alpha}(x)-t_{\alpha}(x)\right)<u\right\} & =\mathbb{E}\left(\widehat{t}_{\alpha}(x)<\tau_{\alpha}(u, x)\right) \\
& =\mathbb{P}\left[\widehat{F}^{x}\left(\tau_{\alpha}(u, x)\right)>\alpha\right] \\
& =\mathbb{P}\left(\widehat{F}^{x}\left(\tau_{\alpha}(u, x)\right)>0\right) .
\end{aligned}
$$


It follows that

$$
\begin{aligned}
\mathbb{P}\left(\widehat{F}^{x}\left(\tau_{\alpha}(u, x)\right)>0\right) \\
\quad=\mathbb{P}\left(0<\widehat{B}_{n}(x, y)+\frac{\widehat{R}_{n}(x, y)}{\widehat{F}_{D}(x)}+\frac{\widehat{Q}_{n}(x, y)}{\widehat{F}_{D}(x)}\right) \\
\quad=\mathbb{P}\left(-\widehat{F}_{D}(x) \widehat{B}_{n}\left(x, \tau_{\alpha}(u, x)\right)-\widehat{R}_{n}\left(x, \tau_{\alpha}(u, x)\right)-\widehat{Q}_{n}\left(x, \tau_{\alpha}(u, x)\right)<0\right) \\
\quad=\mathbb{P}\left(-\widehat{F}_{D}(x) \widehat{B}_{n}\left(x, \tau_{\alpha}(u, x)\right)-\widehat{R}_{n}\left(x, \tau_{\alpha}(u, x)\right)<\widehat{Q}_{n}\left(x, \tau_{\alpha}(u, x)\right)\right) .
\end{aligned}
$$

Thus, the proof of Theorem 1 is a direct consequence of Lemmas 1 4 .

\section{Application to Predictive Interval}

The aim of this section is to construct the confidence band of asymptotic level $(1-\alpha) \%$ for the conditional distribution function estimator where $u_{\alpha / 2}$ is the upper $\alpha / 2$ quantile of standard normal $\mathcal{N}(0,1)$. So that, the following corollary gives us an asymptotic approximation

Corollary 1. By following the above results, for any $x \in \mathcal{F}$ and every $\alpha$, we get

$$
\left[\widehat{F}^{x}(y)-u_{\alpha / 2} \sqrt{\frac{\sigma^{2}(x)}{n \varphi_{n}(x)}}, \widehat{F}^{x}(y)+u_{\alpha / 2} \sqrt{\frac{\sigma^{2}(x)}{n \varphi_{n}(x)}}\right] .
$$

\section{Computational Studies}

The result highlighted in this section is an important investigation of a small numerical study for evaluating the performance of our proposed estimator. More precisely, our main aim is to compare the efficiency of the double-kernel recursive estimation method, to the classical kernel one which has been extensively discussed in the previous many papers. To do that, we consider firstly the following non-parametric model for all $i=1, \ldots, n$

$$
Y_{i}=r\left(X_{i}\right)+\epsilon_{i}
$$

where $\epsilon_{i}$ are random variables independent of $X$ and follow a normal mixture distribution $(1-\lambda) * \mathcal{N}(0,1)+\lambda * \mathcal{N}(4,5)$ and we chose the contamination parameter $\lambda$ to be respectively: $0.1,0.2,0.5,0.7$ and 0.9 with a sample size $\mathrm{n}=100,200$ and 500 . 
In addition, in order to generate the functional variables $\left(X_{i}\right)_{i=1, \ldots, n}$, we use the $R$-routine simul.far of the far package in $R$, This routine simulates a functional autoregressive process with a strong white noise. The simulation experiments used here considered a sinusoidal basis, with five functional axes, of the continuous functions from $[0,1]$ to $\mathbb{R}$. Also, we fix the diagonal matrix $(0.45,0.9,0.34,0.45)$ to define the linear operator with a perturbation coefficient equal to 0.05 . The $X_{i}$ 's curves are discretized in the same grid which is composed of 100 points and are plotted in Figure 1.

Moreover, the response variable $Y_{i}$ is generated from the following operator:

$$
r(x)=5 \int_{0}^{1} \exp (x(t)) d t .
$$

Using this model permits the determination of the theoretical quantile $t_{\alpha}(x)$ such as the conditional distribution of $Y$ given $X=x$ is explicitly given by the distribution of $\epsilon_{i}$ shifted by $r(x)$.

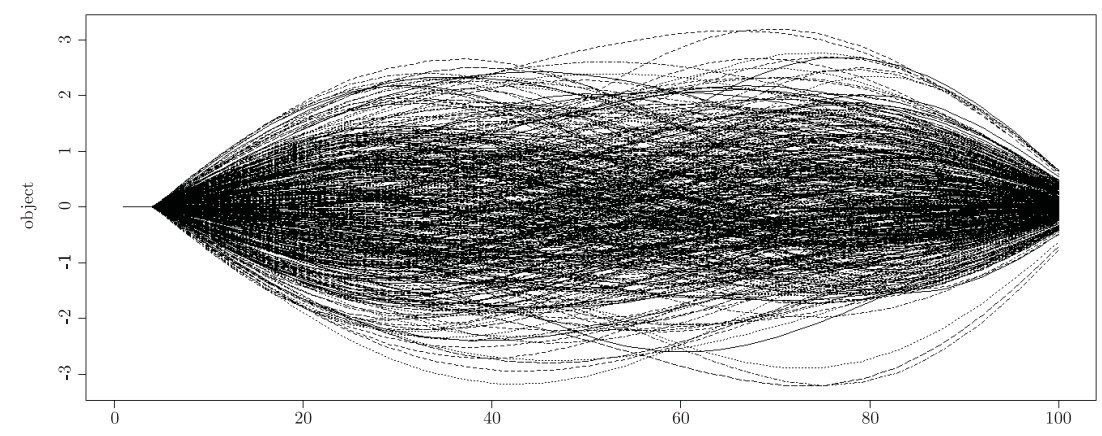

Figure 1: A sample of 100 curves

To give a fair comparison between the two methods we must treat each one under its optimal conditions and specify the different parameters of the both. Unfortunately, to the best of our knowledge, there is no automatic datadriven method available for selecting bandwidths when estimating a conditional quantile function with functional regressors. Thus, for our comparison study we consider a similar bandwidth selector to that used by Ferraty and Vieu [12]. Specifically, the bandwidths $\left(a_{i}, b_{i}\right)$ in the recursive method are 
selected by the following leave-out-one-curve cross-validation procedure

$$
\arg \min _{\left(a_{i}, b_{i}\right) \in A_{n} \times B_{n}} \sum_{j=1}^{n}\left(Y_{j}-\breve{t}_{0.5}^{-j}\left(X_{j}, a_{i}, b_{i}\right)\right)^{2}
$$

where $\breve{t}_{0.5}^{-j}\left(X_{j}, a_{i}, b_{i}\right)$ denotes the double-kernel recursive estimator of the conditional median in the curve $X_{j}$ and computed by the bandwidths $\left(a_{i}, b_{i}\right)$. While for the kernel method we adapt the R-routine named funopare.quantile.lcv. We emphasize that, frequently, we considered a quadratic kernel on $(0,1)$ and the $L^{2}$ metric. Then, we compute the errors to evaluate the performance of these estimators as follows:

- The case of classical double-kernel method, the mean squared error (MSE) is

$$
M S E(D K M)=\frac{1}{n} \sum_{i=1}^{n}\left(\widetilde{t}_{\alpha K M}\left(X_{i}\right)-t_{\alpha}\left(X_{i}\right)\right)^{2}
$$

- The case of recursive double-kernel method, the mean squared error (MSE) is

$$
M S E(R D K M)=\frac{1}{n} \sum_{i=1}^{n}\left(\widetilde{t}_{\alpha}\left(X_{i}\right)-t_{\alpha}\left(X_{i}\right)\right)^{2} .
$$

Therefore, the obtained results of mean squared error are summarized in Table1, Table 2 and Table 3 for the different sample sizes $n=(100,200,500)$, while, Figure 2 simultaneously plots side by side, the estimated conditional quantiles by the RDKM and the ones estimated by the DKM.

Table 1: Mean Squared Error Results for $n=100$.

\begin{tabular}{ccccccc}
\hline & & $\lambda=0.1$ & $\lambda=0.2$ & $\lambda=0.5$ & $\lambda=0.7$ & $\lambda=0.9$ \\
\hline \multirow{3}{*}{$\operatorname{MSE}(\mathrm{DKM})$} & $Q_{1}$ & 4.9110 & 5.4123 & 6.9100 & 7.9121 & 8.9010 \\
& $Q_{2}$ & 2.4420 & 2.9413 & 4.4401 & 5.4410 & 6.4321 \\
& $Q_{3}$ & 4.2511 & 4.7515 & 6.2501 & 1.2552 & 2.2002 \\
\hline \multirow{3}{*}{$\operatorname{MSE}(\mathrm{RDKM})$} & $Q_{1}$ & 2.1302 & 2.3812 & 3.13402 & 3.6305 & 4.1328 \\
& $Q_{2}$ & 1.6920 & 1.9401 & 2.6955 & 3.1921 & 3.6921 \\
& $Q_{3}$ & 2.2924 & 2.5443 & 3.2945 & 3.8421 & 4.3421 \\
\hline
\end{tabular}


Table 2: Mean Squared Error Results for $n=200$.

\begin{tabular}{ccccccc}
\hline & & $\lambda=0.1$ & $\lambda=0.2$ & $\lambda=0.5$ & $\lambda=0.7$ & $\lambda=0.9$ \\
\hline \multirow{3}{*}{$\operatorname{MSE}(\mathrm{DKM})$} & $Q_{1}$ & 4.7801 & 5.2800 & 6.7843 & 7.7736 & 8.7718 \\
& $Q_{2}$ & 2.3110 & 2.8109 & 4.3121 & 5.3102 & 6.3155 \\
& $Q_{3}$ & 4.1220 & 4.6202 & 6.1201 & 1.1201 & 2.0761 \\
\hline \multirow{4}{*}{$\operatorname{MSE}(\mathrm{RDKM})$} & $Q_{1}$ & 2.0030 & 2.2500 & 3.0012 & 3.5050 & 4.0021 \\
& $Q_{2}$ & 1.5610 & 1.8191 & 2.5643 & 3.0667 & 3.5631 \\
& $Q_{3}$ & 2.1609 & 2.4181 & 3.1661 & 3.7108 & 4.2157 \\
\hline
\end{tabular}

Table 3: Mean Squared Error Results for $n=500$.

\begin{tabular}{ccccccc}
\hline & & $\lambda=0.1$ & $\lambda=0.2$ & $\lambda=0.5$ & $\lambda=0.7$ & $\lambda=0.9$ \\
\hline \multirow{3}{*}{$\operatorname{MSE}(\mathrm{DKM})$} & $Q_{1}$ & 4.3900 & 4.8901 & 5.7991 & 7.3865 & 8.3864 \\
& $Q_{2}$ & 1.9210 & 2.4267 & 3.9267 & 4.9200 & 5.9101 \\
& $Q_{3}$ & 3.7373 & 3.7201 & 3.7333 & 0.7343 & 1.6823 \\
\hline \multirow{4}{*}{$\operatorname{MSE}(\mathrm{RDKM})$} & $Q_{1}$ & 1.6101 & 1.8661 & 2.6113 & 2.6670 & 3.6753 \\
& $Q_{2}$ & 1.1702 & 0.9107 & 2.1787 & 2.6708 & 3.1702 \\
& $Q_{3}$ & 1.7777 & 2.0266 & 2.7768 & 3.3252 & 3.8294 \\
\hline
\end{tabular}

Conclusion. Table 1 (respectively Table 2 and Table 3 ) presents the MSE values of both estimates of the quartiles $Q_{1}(\alpha=0.25), Q_{2}(\alpha=0.5)$ and $Q_{3}(\alpha=0.75)$. This simulation involves two interpretations: The first is that Table 1 (respectively Table 2 and Table [3) is clearly showed that the recursive double kernel method gives better results than the classical double kernel method. As can be seen from the Figure 2 as well. The second is that the MSE values increase more substantially (with respect to the value of $\lambda$ ) in the kernel method than in the recursive one.

\section{Appendix}

Proof of Lemma 1. We use the same ideas in Laib and Louani [16]. For all $i=1, \ldots, n$, we start by defining

$$
\eta_{n_{i}}=\left(\frac{\varphi_{n}(x) \gamma_{2}^{2}}{\gamma_{1} \alpha(1-\alpha)}\right)^{1 / 2}\left(H_{i}\left(\tau_{\alpha}\left(t_{\alpha}(x)\right)\right)-F^{x}\left(\tau_{\alpha}\left(t_{\alpha}(x)\right)\right)\right) \frac{K_{i}(x)}{n \varphi_{n}(x)}
$$





Figure 2: Conditional quantiles (Q1, Q2 and Q3) estimation by RDKM (on the left) versus DKM (on the right)

and then $\xi_{n_{i}}=\eta_{n_{i}}-\mathbb{E}\left(\eta_{n_{i}} \backslash \mathfrak{F}_{i-1}\right)$. Subsequently, it is easily seen that

$$
\left(\frac{n \varphi_{n}(x) \gamma_{2}^{2}}{\gamma_{1} \alpha(1-\alpha)}\right)^{1 / 2} \widehat{Q}_{n}\left(x, \tau_{\alpha}\left(t_{\alpha}(x)\right)=\frac{1}{\sqrt{n}} \sum_{i=1}^{n} \xi_{n_{i}}\right.
$$

As $\xi_{n_{i}}$ is a triangular array of martingale differences according the $\sigma$-fields $\mathfrak{F}_{i-1}$, we are in position to apply the central limit theorem based on unconditional Lindeberg condition to prove the asymptotic normality of $\widehat{Q}_{n}(x)$. Thus, it suffices to establish the following two parts:
(a) $\frac{1}{n} \sum_{i=1}^{n} \mathbb{E}\left(\xi_{n_{i}}^{2} \backslash \mathfrak{F}_{i-1}\right) \longrightarrow 1$ in probability;
(b) $\frac{1}{n} \sum_{i=1}^{n} \mathbb{E}\left(\xi_{n_{i}}^{2} \mathbb{I}_{\xi_{n_{i}}^{2}>\epsilon n}\right) \longrightarrow 0$ holds for any $\epsilon>0$ (Lindeberg condition). 
Proof of Part (a). The first part can be easily written as follows

$$
\begin{aligned}
\mathbb{E}\left(\xi_{n_{i}}^{2} \backslash \mathfrak{F}_{i-1}\right) & =\mathbb{E}\left(\left(\eta_{n_{i}}-\mathbb{E}\left(\eta_{n_{i}} \backslash \mathfrak{F}_{i-1}\right)\right)^{2}\right) \\
& =\mathbb{E}\left(\eta_{n_{i}}^{2} \backslash \mathfrak{F}_{i-1}\right)-\mathbb{E}^{2}\left(\eta_{n_{i}} \backslash \mathfrak{F}_{i-1}\right)
\end{aligned}
$$

Thus, we need to prove the validity of the following two statements resulting from (a):

1. $\lim _{n \longrightarrow \infty} \frac{1}{n} \sum_{i=1}^{n} \mathbb{E}^{2}\left(\eta_{n_{i}} \backslash \mathfrak{F}_{i-1}\right)=0_{p}$

2. $\lim _{n \rightarrow \infty} \frac{1}{n} \sum_{i=1}^{n} \mathbb{E}\left(\eta_{n_{i}}^{2} \backslash \mathfrak{F}_{i-1}\right)=1_{p}$

For the first convergence, we have

$$
\begin{aligned}
\mathbb{E}\left(\eta_{n_{i}} \backslash \mathfrak{F}_{i-1}\right)= & \frac{1}{\varphi_{n}(x)}\left(\frac{\varphi_{n}(x) \gamma_{2}^{2}}{\gamma_{1} \alpha(1-\alpha)}\right)^{1 / 2} \mathbb{E}\left[\left[\left(H_{i}\left(\tau_{\alpha}\left(t_{\alpha}(x)\right)\right)\right.\right.\right. \\
& \left.\left.-F^{x}\left(\tau_{\alpha}\left(t_{\alpha}(x)\right)\right) K_{i}(x)\right] \backslash \mathfrak{F}_{i-1}\right] \\
\left|\mathbb{E}\left(\eta_{n_{i}} \backslash \mathfrak{F}_{i-1}\right)\right|= & \frac{1}{\varphi_{n}(x)}\left(\frac{\varphi_{n}(x) \gamma_{2}^{2}}{\gamma_{1} \alpha(1-\alpha)}\right)^{1 / 2} \mid \mathbb{E}\left[\left[\left(\mathbb{E}\left(H_{i}\left(\tau_{\alpha}\left(t_{\alpha}(x)\right)\right) \backslash \mathfrak{B}_{i-1}\right)\right)\right.\right. \\
& \left.\left.-F^{x}\left(\tau_{\alpha}\left(t_{\alpha}(x)\right)\right) K_{i}(x)\right] \backslash \mathfrak{F}_{i-1}\right] \mid \\
= & \frac{1}{\varphi_{n}(x)}\left(\frac{\varphi_{n}(x) \gamma_{2}^{2}}{\gamma_{1} \alpha(1-\alpha)}\right)^{1 / 2} \mid \mathbb{E}\left[\left[\left(\mathbb{E}\left(H_{i}\left(\tau_{\alpha}\left(t_{\alpha}(x)\right)\right) \backslash X_{i}\right)\right)\right.\right. \\
& \left.\left.-F^{x}\left(\tau_{\alpha}\left(t_{\alpha}(x)\right)\right) K_{i}(x)\right] \backslash \mathfrak{F}_{i-1}\right] \mid .
\end{aligned}
$$

Then, under (H1) and (H4), we have:

$$
C \phi_{i}\left(x, a_{i}\right) \leq \mathbb{E}\left(K_{i}(x) \backslash \mathfrak{F}_{i-1}\right) \leq C^{\prime} \phi_{i}\left(x, a_{i}\right)
$$

After that, an integration by parts and a change of variable allow to get

$$
\mathbb{E}\left(H_{i}\left(\left(\tau_{\alpha}\left(t_{\alpha}(x)\right)\right) / X_{i}\right)\right)=\int_{\mathbb{R}} H^{(1)}(t) F^{X_{i}}\left(y-b_{i} t\right) d t
$$


and under (H2), we have

$$
\left|\mathbb{E}\left(H_{i}\left(\tau_{\alpha}\left(t_{\alpha}(x)\right)\right) / X_{i}\right)-F^{x}\left(\tau_{\alpha}\left(t_{\alpha}(x)\right)\right)\right| \leq c a_{i}^{\beta_{1}}+c^{\prime} b_{i}^{\beta_{2}}
$$

combining these results, we have

$$
\left|\mathbb{E}\left(\eta_{n_{i}} \backslash \mathfrak{F}_{i-1}\right)\right| \leq C^{\prime}\left(\frac{\varphi_{n}(x) \gamma_{2}^{2}}{\gamma_{1} \alpha(1-\alpha)}\right)^{1 / 2}\left(c a_{i}^{\beta_{1}}+c^{\prime} b_{i}^{\beta_{2}}\right) \frac{\phi_{i}\left(x, a_{i}\right)}{\varphi_{n}(x)}
$$

So, under (H3), we have

$\frac{1}{n} \sum_{i=1}^{n} \mathbb{E}\left(\eta_{n_{i}} \backslash \mathfrak{F}_{i-1}\right)^{2} \leq C^{\prime 2}\left(\frac{\gamma_{2}^{2}}{\gamma_{1} \alpha(1-\alpha)}\right) \sum_{i=1}^{n}\left(c a_{i}^{\beta_{1}}+c^{\prime} b_{i}^{\beta_{2}}\right)^{2} \frac{\phi_{i}^{2}\left(x, a_{i}\right)}{n \varphi_{n}(x)}=o_{p}(1)$

Now, we treat the convergence (2). Indeed, we observe that

$$
\begin{aligned}
& \frac{1}{n} \sum_{i=1}^{n}\left(\mathbb{E}\left(\eta_{n_{i}}^{2} \backslash \mathfrak{F}_{i-1}\right)\right) \\
&=\frac{1}{n \varphi_{n}^{2}(x)}\left(\frac{\varphi_{n}(x) \gamma_{2}^{2}}{\gamma_{1} \alpha(1-\alpha)}\right) \sum_{i=1}^{n} \mathbb{E}\left[\left(H_{i}\left(\tau_{\alpha}\left(t_{\alpha}(x)\right)\right)-F^{x}\left(\tau_{\alpha}\left(t_{\alpha}(x)\right)\right)\right)^{2} K_{i}^{2}(x) \backslash \mathfrak{F}_{i-1}\right] \\
&=\frac{1}{n \varphi_{n}^{2}(x)}\left(\frac{\varphi_{n}(x) \gamma_{2}^{2}}{\gamma_{1} \alpha(1-\alpha)}\right) \sum_{i=1}^{n}\left[\mathbb{E}\left(H_{i}^{2}\left(\tau_{\alpha}\left(t_{\alpha}(x)\right)\right) K_{i}^{2}(x) \backslash \mathfrak{F}_{i-1}\right)\right. \\
& \quad-2 F^{x}\left(\tau_{\alpha}\left(t_{\alpha}(x)\right)\right) \mathbb{E}\left(H_{i}\left(\tau_{\alpha}\left(t_{\alpha}(x)\right)\right) K_{i}^{2}(x) \backslash \mathfrak{F}_{i-1}\right) \\
&\left.+\left(F^{x}\left(\tau_{\alpha}\left(t_{\alpha}(x)\right)\right)\right)^{2} \mathbb{E}\left(K_{i}^{2}(x) \backslash \mathfrak{F}_{i-1}\right)\right] .
\end{aligned}
$$

We put:

$$
\begin{aligned}
I_{1} & =\sum_{i=1}^{n} \mathbb{E}\left(H_{i}^{2}\left(\tau_{\alpha}\left(t_{\alpha}(x)\right)\right) K_{i}^{2}(x) \backslash \mathfrak{F}_{i-1}\right) \\
I_{2} & =\sum_{i=1}^{n} \mathbb{E}\left(H_{i}\left(\tau_{\alpha}\left(t_{\alpha}(x)\right)\right) K_{i}^{2}(x) \backslash \mathfrak{F}_{i-1}\right) \\
I_{3} & =\sum_{i=1}^{n} \mathbb{E}\left(K_{i}^{2}(x) \backslash \mathfrak{F}_{i-1}\right) .
\end{aligned}
$$


We write:

$$
\begin{aligned}
I_{1}= & F^{x}\left(\tau_{\alpha}\left(t_{\alpha}(x)\right)\right) \sum_{i=1}^{n} \mathbb{E}\left[K_{i}^{2}(x) \backslash \mathfrak{F}_{i-1}\right] \\
& \left.+\sum_{i=1}^{n} \mathbb{E}\left[H_{i}^{2}\left(\tau_{\alpha}\left(t_{\alpha}(x)\right)\right) K_{i}^{2}(x) \backslash \mathfrak{F}_{i-1}\right)\right] \\
& -F^{x}\left(\tau_{\alpha}\left(t_{\alpha}(x)\right)\right) \sum_{i=1}^{n} \mathbb{E}\left[K_{i}^{2}(x) \backslash \mathfrak{F}_{i-1}\right] \\
= & F^{x}\left(\tau_{\alpha}\left(t_{\alpha}(x)\right)\right) \sum_{i=1}^{n} \mathbb{E}\left[K_{i}^{2}(x) \backslash \mathfrak{F}_{i-1}\right] \\
& \left.+\sum_{i=1}^{n} \mathbb{E}\left[\left(\mathbb{E}\left(H_{i}^{2}\left(\tau_{\alpha}\left(t_{\alpha}(x)\right)\right) \backslash X_{i}\right) K_{i}^{2}(x)\right) \backslash \mathfrak{F}_{i-1}\right)\right] \\
& -F^{x}\left(\tau_{\alpha}\left(t_{\alpha}(x)\right)\right) \sum_{i=1}^{n} \mathbb{E}\left[K_{i}^{2}(x) \backslash \mathfrak{F}_{i-1}\right] \\
\leq & \left.\sum_{i=1}^{n} \mathbb{E}\left[\left(\mathbb{E}\left(H_{i}^{2}\left(\tau_{\alpha}\left(t_{\alpha}(x)\right)\right) \backslash X_{i}\right) K_{i}^{2}(x)\right) \backslash \mathfrak{F}_{i-1}\right)\right] \\
& -F^{x}\left(\tau_{\alpha}\left(t_{\alpha}(x)\right)\right) \sum_{i=1}^{n} \mathbb{E}\left[K_{i}^{2}(x) \backslash \mathfrak{F}_{i-1}\right] .
\end{aligned}
$$

Using the same argument as those used in proof of part (a), then we have:

$$
\frac{1}{n \varphi_{n}\left(x, a_{i}\right)} I_{2}=\circ(1)
$$

For $I_{3}$, we use the same ideas in Ferraty et al (2009) to get:

$$
\mathbb{E}\left[K_{i}^{2}(x) \backslash \mathfrak{F}_{i-1}\right]=K^{2}(1) \phi_{i}\left(x, a_{i}\right)-\int_{0}^{1}\left(K^{2}(u)\right)^{\prime} \phi_{i}\left(x, u a_{i}\right) d u
$$

and

$$
\mathbb{E}\left[K_{1}^{2}(x) \backslash \mathfrak{F}_{i-1}\right]=K(1) \phi_{i}\left(x, a_{i}\right)-\int_{0}^{1}(K(u))^{\prime} \phi_{i}\left(x, u a_{i}\right) d u
$$

so under (H1), we have:

$$
\frac{1}{n \varphi\left(x, a_{i}\right)} \sum_{i=1}^{n} \mathbb{E}\left[K_{i}^{2}(x) \backslash \mathfrak{F}_{i-1}\right]
$$




$$
\begin{aligned}
& =\frac{K^{2}(1)}{n \varphi\left(x, a_{i}\right)} \sum_{i=1}^{n} \phi_{i}\left(x, a_{i}\right)-\int_{0}^{1}\left(K^{2}(u)\right)^{\prime} \frac{1}{n \varphi\left(x, a_{i}\right)} \sum_{i=1}^{n} \phi_{i}\left(x, u a_{i}\right) d u \\
& =K^{2}(1)-\int_{0}^{1}\left(K^{2}(u)\right)^{\prime} \beta_{x}(u) d u+o_{p}(1)=\gamma_{2}+o_{p}(1) .
\end{aligned}
$$

and

$$
\frac{1}{n \varphi\left(x, a_{i}\right)} \mathbb{E}\left[K_{1}(x) \backslash \mathfrak{F}_{i-1}\right]=\gamma_{1}+o_{p}(1)
$$

we deduce that $\lim _{n \rightarrow \infty} \sum_{i=1}^{n} \mathbb{E}\left(\eta_{n_{i}}^{2} \backslash \mathfrak{F}_{i-1}\right)=1$ which complete the proof of part (a).

Proof of Part (b). The Lindeberg condition in show which implies that

$$
\mathbb{E}\left[\xi_{n_{i}}^{2} \mathbb{I}_{\xi_{n_{i}}>n \epsilon}\right] \leq 4 \mathbb{E}\left[\eta_{n_{i}}^{2} \mathbb{I}_{\eta_{n_{i}}>n \epsilon / 2}\right]
$$

Let $a>1$ and $b>1$ such that $\frac{1}{a}+\frac{1}{b}=1$. Making use now the Hölder's and Markov's inequalities one can write for all $\varepsilon>0$

$$
\mathbb{E}\left[\eta_{n_{i}}^{2} \mathbb{I}_{\eta_{n_{i}}>n \epsilon / 2}\right] \leq \frac{\mathbb{E}\left(\eta_{n_{i}}\right)^{2 a}}{(n \epsilon / 2)^{2 a / b}}
$$

Taking $C_{0} \in \mathbb{R}_{+}^{*}$ and $2 a=2+\delta$ form some $\delta>0$, such that $\mathbb{E}\left(\left|Y_{i}\right|^{2+\delta}\right)$ $<\infty$ and $\mathbb{E}\left(\left|H_{i}-F^{x}\right|^{2+\delta} \backslash X_{i}=u\right)=\bar{W}_{2+\delta}(u)$ is a continuous function, to get the following

$4 \mathbb{E}\left[\eta_{n_{i}}^{2} \mathbb{I}_{\eta_{n_{i}}>n \epsilon}\right]$

$$
\begin{aligned}
& \leq C_{0}\left(\frac{\varphi_{n}\left(x, a_{i}\right) \gamma_{1}^{2}}{\gamma_{2}(\alpha(1-\alpha))}\right)^{(2+\delta)} \frac{1}{\left(\varphi_{n}\left(x, a_{i}\right)\right)^{2+\delta}}\left[\left(\left|H_{i}-F^{x}\right|^{2+\delta} K_{i}^{2+\delta}(x)\right)^{2+\delta}\right] \\
& \leq C_{0}\left(\frac{\varphi_{n}\left(x, a_{i}\right) \gamma_{1}^{2}}{\gamma_{2}(\alpha(1-\alpha))}\right)^{(2+\delta)} \frac{1}{\left(\varphi_{n}\left(x, a_{i}\right)\right)^{2+\delta}} \mathbb{E}\left[\mathbb{E}\left[\left|H_{i}-F^{x}\right|^{2+\delta} K_{i}^{2+\delta}(x) \backslash X_{i}\right]\right] \\
& \leq C_{0}\left(\frac{\varphi_{n}\left(x, a_{i}\right) \gamma_{1}^{2}}{\gamma_{2}(\alpha(1-\alpha))}\right)^{(2+\delta)} \frac{1}{\left(\varphi_{n}\left(x, a_{i}\right)\right)^{2+\delta}} \mathbb{E}\left[K_{i}^{2+\delta}(x) \bar{W}_{2+\delta}\left(X_{i}\right)\right] \\
& \leq C_{0}\left(\frac{\varphi_{n}\left(x, a_{i}\right) \gamma_{1}^{2}}{\gamma_{2}(\alpha(1-\alpha))}\right)^{(2+\delta)} \frac{1}{\left(\varphi_{n}\left(x, a_{i}\right)\right)^{2+\delta}} \mathbb{E}\left[K_{i}^{2+\delta}(x)\left|\bar{W}_{2+\delta}\left(X_{i}\right)-\bar{W}_{2+\delta}(x)\right|\right.
\end{aligned}
$$




$$
\begin{aligned}
& \left.+\left|\bar{W}_{2+\delta}(x) \mathbb{E}\left(K_{i}^{2+\delta}(x)\right)\right|\right] \\
\leq & C_{0}\left(\frac{\gamma_{1}^{2}}{\gamma_{2}(\alpha(1-\alpha))}\right)^{(2+\delta)}\left(\mathbb{E}\left(K_{i}^{2+\delta}(x)\right)\left(\left|\bar{W}_{2+\delta}(x)\right|+\circ(1)\right)\right)
\end{aligned}
$$

Consequently $\frac{1}{n} \sum_{i=1}^{n} \mathbb{E}\left[\xi_{n_{i}}^{2} \mathbb{I}_{\xi_{n_{i}}>n \epsilon}\right] \longrightarrow 0$ as $n \longrightarrow \infty$ which complete the proof of lemma.

Proof of Lemma 2, Observe that:

$$
\begin{aligned}
\widehat{F}_{D}(x)-1= & \frac{1}{\varphi_{n}\left(x, a_{i}\right)} \sum_{i=1}^{n}\left[\left[K_{i}(x)-\mathbb{E}\left(K_{i}(x) \backslash \mathfrak{F}_{i-1}\right)\right]\right. \\
= & \underbrace{\frac{1}{\varphi_{n}\left(x, a_{i}\right)} \sum_{i=1}^{n}\left[K_{i}(x)-\mathbb{E}\left(K_{i}(x) \backslash \mathfrak{F}_{i-1}\right)\right]}_{T_{1}} \\
& +\underbrace{\left.\left.\frac{1}{\varphi_{n}\left(x, a_{i}\right)} \sum_{i=1}^{n}\left[K_{i}(x) \backslash \mathfrak{F}_{i-1}\right)\right]-1\right]}_{T_{2}}
\end{aligned}
$$

The proof of the lemma follows then if show that:

1. $T_{1}=\circ(1)$ as $n \longrightarrow \infty$;

2. $T_{2} \longrightarrow 0$ in probability as $n \longrightarrow \infty$

for $T_{2}$, and under $(\mathrm{H} 1)$ and $(\mathrm{H} 3)$ we prove that:

$$
\frac{1}{\varphi_{n}\left(x, a_{i}\right)} \sum_{i=1}^{n}\left[\mathbb{E}\left(K_{i}(x) \backslash \mathfrak{F}_{i-1}\right)\right]=\circ(1) \text { as } n \longrightarrow \infty
$$

So, it is easily seen that

$$
T_{2} \longrightarrow 0 \text { in probability as } n \longrightarrow \infty \text {. }
$$

For the first term $T_{1}$, observe that $T_{1}(x)=\sum_{i=1}^{n} L_{n_{i}}(x)$ where $\left\{L_{n_{i}}(x)\right\}$ is a triangular array of martingale differences with respect to the $\sigma-$ field $\mathfrak{F}_{i-1}$. 
Combining next the Burkholder inequality (see P. H. All and C. Heyde p(23), 1980) and Jensen inequality (see Laib and Louani 2011), we obtain for any $\epsilon>0$, there exist a constant $C_{0}>0$ such that

$$
\mathbb{P}\left(\left|T_{1}\right|>\epsilon\right) \leq C_{0} \frac{\mathbb{E}\left(K_{1}^{2}(x)\right)}{\epsilon^{2} n\left(\varphi_{n}\left(x, a_{i}\right)\right)^{2}}=\circ\left(\frac{1}{\epsilon^{2} n \varphi_{n}(x)}+\circ(1)\right)
$$

Since $n \varphi_{n}(x) \longrightarrow \infty$, we conclude then that $T_{1}(x)=\circ(1)$ in probability as $n \longrightarrow \infty$ which completes the proof of Lemma 2.

Proof of Lemma 3. We have

$$
\widehat{B}_{n}\left(x, \tau_{\alpha}\left(t_{\alpha}(x)\right)\right)=\frac{\bar{F}_{N}\left(x, \tau_{\alpha}\left(t_{\alpha}(x)\right)\right)}{\bar{F}_{D}\left(x, \tau_{\alpha}\left(t_{\alpha}(x)\right)\right)} .
$$

We write

$$
\begin{aligned}
\left|\widehat{B}_{n}\left(x, \tau_{\alpha}\left(t_{\alpha}(x)\right)\right)\right| & \frac{1}{\sum_{i=1}^{n} \mathbb{E}\left(K_{i}(x) \backslash \mathfrak{F}_{i-1}\right)} \sum_{i=1}^{n}\left[\mathbb{E}\left[K_{i}(x) \mathbb{E}\left[H_{i}\left(\tau_{\alpha}\left(t_{\alpha}(x)\right)\right) \backslash \mathfrak{B}_{i-1}\right] \backslash \mathfrak{F}_{i-1}\right]\right. \\
& \left.-F^{x}\left(\tau_{\alpha}\left(t_{\alpha}(x)\right)\right) \mathbb{E}\left[K_{i}(x) \backslash \mathfrak{F}_{i-1}\right]\right] \\
= & \frac{1}{\sum_{i=1}^{n} \mathbb{E}\left(K_{i}(x) \backslash \mathfrak{F}_{i-1}\right)} \sum_{i=1}^{n}\left[\mathbb{E}\left[K_{i}(x) \mathbb{E}\left[H_{i}\left(\tau_{\alpha}\left(t_{\alpha}(x)\right)\right) \backslash X_{i}\right] \backslash \mathfrak{F}_{i-1}\right]\right. \\
& \left.-F^{x}\left(\tau_{\alpha}\left(t_{\alpha}(x)\right)\right) \mathbb{E}\left[K_{i}(x) \backslash \mathfrak{F}_{i-1}\right]\right] \\
\leq & \frac{1}{\sum_{i=1}^{n} \mathbb{E}\left(K_{i}(x) \backslash \mathfrak{F}_{i-1}\right)} \sum_{i=1}^{n} \mathbb{E}\left[K_{i}(x) \mid \mathbb{E}\left[H_{i}\left(\tau_{\alpha}\left(t_{\alpha}(x)\right)\right) \backslash X_{i}\right]\right. \\
& \left.-F^{x}\left(\tau_{\alpha}\left(t_{\alpha}(x)\right)\right) \mid \backslash \mathfrak{F}_{i-1}\right]
\end{aligned}
$$

Next, an integration by parts and change of variable allow to get:

$$
\mathbb{E}\left[H_{i}\left(\tau_{\alpha}\left(t_{\alpha}(x)\right)\right) \backslash X_{i}\right]=\int_{\mathbb{R}} H^{(1)}(t) F^{X_{i}}\left(\tau_{\alpha}\left(t_{\alpha}(x)\right)-b_{i} t\right) d t
$$


Thus, we have

$$
\begin{aligned}
\mid \mathbb{E} & {\left[H_{i}\left(\tau_{\alpha}\left(t_{\alpha}(x)\right)\right) \backslash X_{i}\right]-F^{x}\left(\tau_{\alpha}\left(t_{\alpha}(x)\right)\right) \mid } \\
& \leq \int_{\mathbb{R}} H^{(1)}(t)\left|F^{X_{i}}\left(\left(\tau_{\alpha}\left(t_{\alpha}(x)\right)\right)-b_{i} t\right)-F^{x}\left(\tau_{\alpha}\left(t_{\alpha}(x)\right)\right)\right| d t
\end{aligned}
$$

Under (H2), we obtain that

$$
\begin{aligned}
& \mathbb{I}_{B\left(x, a_{i}\right)}\left(X_{i}\right)\left|\mathbb{E}\left[H_{i}\left(\tau_{\alpha}\left(t_{\alpha}(x)\right)\right) \backslash X_{i}\right]-F^{x}\left(\tau_{\alpha}\left(t_{\alpha}(x)\right)\right)\right| \\
& \quad \leq C \int_{\mathbb{R}} H^{(1)}(t)\left(a_{i}^{\beta_{1}}+|t|^{\beta_{2}} b_{i}^{\beta_{2}}\right) d t
\end{aligned}
$$

and under (H4) we prove that:

$$
\frac{1}{n} \sum_{i=1}^{n} \mathbb{E}\left[K_{i}(x) \backslash \mathfrak{F}_{i-1}\right]=\circ(1)
$$

by combining the statements (5) and (6) we achieve the proof of our lemma.

Proof of Lemma 4. We put $t=\tau_{\alpha}\left(t_{\alpha}(x)\right)$ and we write

$$
\begin{aligned}
\widehat{R}_{n}(x, t) & =-\left(\widehat{B}_{n}(x, t)-F^{x}(t)\right)\left(\widehat{F}_{N}(x, t)-\bar{F}_{N}(x, t)\right) \\
& =-\left(\frac{\bar{F}_{N}(x, t)-F^{x}(t) \bar{F}_{D}(x, t)}{\bar{F}_{D}(x, t)}\right)\left(\widehat{F}_{N}(x, t)-\bar{F}_{N}(x, t)\right) .
\end{aligned}
$$

Clearly, it suffices to show that:
1. $\left(\frac{\bar{F}_{N}(x, t)-F^{x}(t) \bar{F}_{D}(x, t)}{\bar{F}_{D}(x, t)}\right)=\circ(1)$,
2. $\left(\widehat{F}_{N}(x, t)-\bar{F}_{N}(x, t)\right)=\circ(1)$.

The proof of the first part uses arguments similar to those used in the proof of Lemma 3. While the second part will be established if these two following insertions are checked
(i) $\mathbb{E}\left(\widehat{F}_{N}(x, t)-\bar{F}_{N}(x, t)\right)=0$,
(ii) $\operatorname{var}\left(\widehat{F}_{N}(x, t)-\bar{F}_{N}(x, t)\right) \longrightarrow 0$ as $n \longrightarrow \infty$. 
For all $i=1, \cdots, n$, we put

$$
\triangle_{i}(x, t)=\frac{1}{n \varphi_{n}\left(x, a_{i}\right)}\left[K_{i}(x) H_{i}(t)-\mathbb{E}\left[K_{i}(x) H_{i}(t) \backslash \mathfrak{F}_{i-1}\right]\right]
$$

where $\triangle_{i}(x, t)$ is a triangular array of martingale differences according to the $\sigma-$ fields $\mathfrak{F}_{i-1}$, next by (H1)(ii) and (H4) we obtain

$$
\widehat{F}_{N}(x, t)-\bar{F}_{N}(x, t)=\sum_{i=1}^{n} \triangle_{i}(x, t)
$$

$\mathbb{E}\left(\triangle_{i}(x, t)\right)=0$ by definition of $\triangle_{i}(x, t)$.

For (ii), we have by Burkholder's inequality

$$
\mathbb{E}\left[\sum_{i=1}^{n}\left(\triangle_{i}(x, t)\right)\right]^{2} \leq \sum_{i=1}^{n} \mathbb{E}\left[\triangle_{i}^{2}(x, t)\right]
$$

Furthermore, by Jensen's inequality we have:

$$
\begin{aligned}
\mathbb{E}\left[\triangle_{i}^{2}(x, t)\right] & \leq \frac{1}{\left(n \varphi_{n}\left(x, a_{i}\right)\right)^{2}} \mathbb{E}\left[K_{i}^{2}(x) H_{i}^{2}(t) \backslash \mathfrak{F}_{i-1}\right] \\
& \leq \frac{1}{\left(n \varphi_{n}\left(x, a_{i}\right)\right)^{2}} \mathbb{E}\left[K_{i}^{2}(x) \backslash \mathfrak{F}_{i-1}\right] \\
& \leq \frac{1}{\left(n \varphi_{n}\left(x, a_{i}\right)\right)^{2}} \mathbb{P}\left(X_{i} \in B\left(x, a_{i}\right) \backslash \mathfrak{F}_{i-1}\right) \\
& \leq \frac{1}{\left(n \varphi_{n}\left(x, a_{i}\right)\right)^{2}} \phi_{i}\left(x, a_{i}\right)
\end{aligned}
$$

Likewise, we get

$$
\sum_{i=1}^{n} \mathbb{E}\left[\triangle_{i}^{2}(x, t)\right] \leq \frac{\sum_{i=1}^{n} \phi_{i}\left(x, a_{i}\right)}{n^{2} \varphi_{n}^{2}\left(x, a_{i}\right)}
$$

Thence, since (H1)(ii) is verified, we deduce that

$$
\operatorname{var}\left(\widehat{F}_{N}(x, t)-\bar{F}_{N}(x, t)\right) \longrightarrow 0 \text { as } n \longrightarrow \infty .
$$




\section{Acknowledgments}

The authors would like to thank the referees for their constructive criticisms and suggestions that have led to improve the representation of this paper.

\section{References}

1. A. Amiri, Estimateurs fonctionnels récursifs et leurs applications à la prévision, Doctoral Thesis, the University of Avignon and the Pays de Vaucluse, 2010.

2. A. Amiri, C. Crambes and B. Thiam, Recursive estimation of nonparametric regression with functional covariate, Computational Statistics and Data Analysis, 69 (2014), 154172.

3. F. Benziadi, A. Laksaci and F. Tebboune, Recursive kernel estimate of the conditional quantile for functional ergodic data, Communications in Statistics-Theory and Methods 45(2016), No.11, 3097-3113.

4. A. Bouadjemi, Asymptotic normality of the recursive kernel estimate of conditional cumulative distribution function, J. Probab. Stat. Sci., 12 (2014), No.@, 117-126.

5. H. Cardot, C. Crambes and P. Sarda, Quantile regression when the covariates are functions, Nonparametric Statistics 17 (2005), No.7, 841-856.

6. H. Cardot, P. Cnac, P. A. Zitt et al., Recursive estimation of the conditional geometric median in hilbert spaces, Electronic Journal of Statistics 6 (2012), 2535-2562.

7. M. Chaouch and S. Khardani, Randomly censored quantile regression estimation using functional stationary ergodic data, Journal of Nonparametric Statistics 27 (2015), No.1, 65-87.

8. S. Dabo-Niang and A. Laksaci, Nonparametric estimation of conditional quantiles when the regressor is valued in a semi-metric space, 2008.

9. M. Ezzahrioui and E. Ould-Saïd, Asymptotic normality of the kernel estimator of conditional quantiles in a normed space, Far East Journal of Theoretical Statistics $\mathbf{2 5}$ (2008a), 15-38.

10. M. Ezzahrioui and E. Ould-Saïd, Asymptotic results of a nonparametric conditional quantile estimator for functional time series, Communications in Statistics, Theory and Methods 37(2008b), No.17, 2735-2759.

11. F. Ferraty, A. Laksaci and P. Vieu, Estimating some characteristics of the conditional distribution in nonparametric functional models, Statistical Inference for Stochastic Processes 9 (2006), No.1, 47-76.

12. F. Ferraty and P. Vieu, Nonparametric functional data analysis, theory and practice, Springer Science and Business Media (2006).

13. A. Gannoun, J. Saracco and K. Yu, Nonparametric prediction by conditional median and quantiles, Journal of Statistical Planning and inference 117 (2003), No.2, 207-223. 
14. R. Koenker, Quantile regression, Econometric Society Monograph Series, 2005.

15. R. Koenker and G. Bassett, Regression quantiles, Econometrika, 46 (1978), 3350, Mathematical Reviews (MathSciNet): MR474644 Digital Object Identifier: doi, 10:1913643.

16. N. Laib and D. Louani, Nonparametric kernel regression estimation for functional stationary ergodic data: asymptotic properties, Journal of Multivariate Analysis, 101 (2010), No.10, 2266-2281.

17. A. Laksaci, M. Lemdani and E. Ould-Saïd, A generalized 11-approach for a kernel estimator of conditional quantile with functional regressors: Consistency and asymptotic normality, Statistics and Probability Letters, 79 (2009), No.8, 1065-1073.

18. E. Masry, Nonparametric regression estimation for dependent functional data: asymptotic normality, Stochastic Processes and their Applications, 115 (2005), 155-177.

19. E. Masry and L. Györfi, Strong consistency and rates for recursive probability density estimators of stationary processes, Journal of Multivariate Analysis, 22 (1987), No.1, 79-93.

20. C. J. Stone, Consistent nonparametric regression, The Annals of Statistics, (1977), 595-620.

21. C. T. Wolverton and T. J. Wagner, Recursive estimates of probability densities, IEEE Transactions on Systems Science and Cybernetics, 5 (1969), No.3, 246-247.

22. L. Zhengyan and L. Degui, Asymptotic normality for $L_{1}$-norm kernel estimator of conditional median under association dependence, Journal of Multivariate Analysis 98 (2007), 1214-1230. 